\title{
Families of disjoint divisors on varieties
}

\author{
Fedor A. Bogomolov ${ }^{1,2}$. Alena Pirutka ${ }^{1}$. \\ Aaron Michael Silberstein ${ }^{3}$
}

Received: 21 April 2015 / Revised: 16 January 2016 / Accepted: 27 May 2016 /

Published online: 5 July 2016

(C) Springer International Publishing AG 2016

\begin{abstract}
Following the work of Totaro and Pereira, we study sufficient conditions under which collections of pairwise-disjoint divisors on a variety over an algebraically closed field are contained in the fibers of a morphism to a curve. We prove that $\rho_{\mathrm{W}}(X)+1$ pairwise-disjoint, connected divisors suffice for proper, normal varieties $X$, where $\rho_{\mathrm{w}}(X)$ is a modification of the Néron-Severi rank of $X$ (they agree when $X$ is projective and smooth). We then prove a strong counterexample in the affine case: if $X$ is quasi-affine and of dimension $\geqslant 2$ over a countable, algebraically-closed field $k$, then there exists a (countable) collection of pairwise-disjoint divisors which cover the $k$-points of $X$, so that for any non-constant morphism from $X$ to a curve, at most finitely many are contained in the fibers thereof. We show, however, that an
\end{abstract}

\footnotetext{
The first author acknowledges that the article was prepared within the framework of a subsidy granted to the HSE by the Government of the Russian Federation for the implementation of the Global Competitiveness Program. The first author was also supported by a Simons Travel Grant. The second author thanks the University of Chicago and the Embassy of France in United States for their support for a short-term visit to the University of Chicago in April, 2015. The third author gratefully acknowledges the support of NSF Grant DMS-1400683. This paper was conceived at Workshop \# 1444 at Oberwolfach, and we gratefully acknowledge the hospitality of the Matematisches Forschungsinstitut Oberwolfach.
}

$凶$ Aaron Michael Silberstein

asilbers@math.uchicago.edu

Fedor A. Bogomolov

bogomolo@cims.nyu.edu

Alena Pirutka

pirutka@cims.nyu.edu

1 Courant Institute of Mathematical Sciences, New York University, 251 Mercer Str., New York, NY 10012, USA

2 Laboratory of Algebraic Geometry, NRU HSE, 7 Vavilova Str., Moscow 117312, Russia

3 The University of Chicago, 5734 S. University Ave., Chicago, IL 60637, USA 
uncountable collection of pairwise-disjoint, connected divisors in any normal variety over an algebraically-closed field must be contained in the fibers of a morphism to a curve.

Keywords Disjoint divisors · Regular functions · Hodge index theorem · Chow group $\cdot$ Geometric reconstruction

Mathematics Subject Classification $14 \mathrm{C} 05 \cdot 14 \mathrm{C} 25 \cdot 14 \mathrm{D} 05$

\section{Introduction}

The goal of this note is to give a set-theoretic condition under which collections of pairwise-disjoint divisors on varieties over an algebraically-closed field are contained in the fibers of a single morphism to a curve. We first adapt the methods of Totaro [15] and Pereira [12] to produce a stronger bound in the projective, smooth case in characteristic zero, and we generalize these results to normal, proper varieties in all characteristics.

We obtain the following result, which generalizes the theorems of Totaro and Pereira, loc. cit., to normal, proper varieties of arbitrary characteristic. Here, $\rho_{\mathrm{W}}(X)$ is an invariant of the variety $X$, equal to the Néron-Severi rank when $X$ is smooth and projective, and finite in all cases.

Theorem 1.1 Let X be a normal, proper, integral variety defined over an algebraically closed field $k$. Let $\left\{D_{i}\right\}_{i \in I}$ be a collection of pairwise-disjoint, reduced, codimensionone, connected subvarieties of $X$. Assume that $\# I \geqslant \rho_{\mathrm{w}}(X)+1$. Then there is a smooth, projective curve $C$ and a surjective morphism $f: X \rightarrow C$ with connected fibers such that for any $i \in I$, the divisor $D_{i}$ is contained in a fiber of $f$. Furthermore, there is a set $\Sigma \subseteq I$ so that $\# \backslash \Sigma \leqslant \rho_{\mathrm{w}}(X)-2$ and for each $i \in \Sigma, D_{i}$ is equal (set-theoretically) to a fiber of $f$.

In Pereira and Totaro's approaches - which work only in the smooth case-\# I must be at least $\rho_{\mathrm{w}}(X)+2$; our extra saving comes from an extra application of the Hodge index theorem.

In the affine case we have the following explicit counterexample.

Theorem 1.2 Let $\mathbb{A}_{k}^{2}$ be the affine plane over a countable, algebraically-closed field $k$. Then there is a countable family $\left\{D_{i}\right\}_{i \in I}$ of integral, Zariski-closed, codimension-one subvarieties of $X$, such that:

- The divisors $D_{i}$ are pairwise disjoint and their $k$-points cover $\mathbb{A}^{2}$, i.e. $\mathbb{A}^{2}(k)=$ $\bigcup_{i \in I} D_{i}(k)$.

- For any non-constant morphism $f: \mathbb{A}^{2} \rightarrow C$ to a curve, at most finitely many of $D_{i}$ are contained in fibers of $f$.

As a corollary, we easily deduce

Theorem 1.3 Let $X$ be a quasi-affine variety over a countable, algebraically-closed field $k$. Then there is a countable family $\left\{D_{i}\right\}_{i \in I}$ of connected, Zariski-closed, codimension-one subvarieties of $X$, such that: 
- The divisors $D_{i}$ are pairwise disjoint and their $k$-points cover $X$, i.e., $X(k)=$ $\bigcup_{i \in I} D_{i}(k)$.

- For any non-constant morphism $f: X \rightarrow C$, at most finitely many of the $D_{i}$ are contained in fibers of $f$.

We can salvage this counterexample if $I$ is uncountable.

Theorem 1.4 Let $X$ be any normal variety over an algebraically-closed field $k$, and let $\left\{D_{i}\right\}_{i \in I}$ be an uncountable collection of pairwise-disjoint, reduced, codimensionone, connected subvarieties of $X$. Then there is a normal curve $C$ and a non-constant morphism $f: X \rightarrow C$ with connected fibers so that each $D_{i}$ is contained in a fiber of $f$.

In particular, if a set of divisors covers the $k$-points of the variety when $k$ is uncountable, there are uncountably many divisors.

This paper provides a tool to approach the third author (A.S.)'s program of Geometric Reconstruction [13] in the first author (F.B.)'s program of Birational Anabelian Geometry. In Bogomolov's program, we take a field $K$ which is the function field of an algebraic variety $X$ of dimension $\geqslant 2$ defined over an algebraically-closed field, and the goal is to reconstruct $K$ from its absolute Galois group $G_{K}$. In Geometric Reconstruction, the goal is to reconstruct individual varieties with a given function field $K$ as group-theoretically defined objects in $G_{K}$. The results in this paper are crucial in an upcoming paper by the third author in proving geometric reconstruction for function fields $K$ of transcendence degree 2 over $\overline{\mathbb{Q}}$ from the maximal, 2-step nilpotent, pro- $\ell$ quotient of $G_{K}$.

\section{Disjoint divisors on proper varieties}

In this section we prove Theorem 1.1, following the proof of Totaro [15], but with two additional arguments. Totaro and Pereira prove the theorem in characteristic zero, for smooth, projective varieties. First, in order to generalize to characteristic $p$, we reduce the theorem for $X$ normal and projective to the case of a general surface which is an intersection of hyperplane sections; this allows us to use resolution of singularities of surfaces, in arbitrary characteristic. By appealing to the Hodge index theorem we reduce the number of pairwise-disjoint divisors to the theoretical minimum.

\subsection{Divisors and Albanese varieties}

We begin with the notion of divisor class group we will use throughout the paper. Recall that for a variety $Y$ over a field $k$ we denote by $Z^{1}(Y)$ the group of Weil prime divisors on $X$-that is, finite, linear combinations of closed, integral, codimension-one subvarieties and by $\mathrm{CH}^{1}(Y)$ the quotient of $Z^{1}(Y)$ by linear equivalence.

Let $X$ be a normal projective integral variety defined over an algebraically closed field $k$ and $U \subset X$ be the smooth locus of $X$. Fix a prime $\ell$ not equal to the characteristic of $k$. Since $X$ is normal, the singular locus is codimension $\geqslant 2$ and the restriction map $\mathrm{CH}^{1}(X) \rightarrow \mathrm{CH}^{1}(U)$ is an isomorphism. Since $U$ is smooth, we have the cycle class 
map $\mathrm{CH}^{1}(U) \rightarrow H_{\text {et }}^{2}\left(U, \mathbb{Q}_{\ell}(1)\right)[4,2.1 .1]$. We then denote by $\mathrm{CH}^{1}(X)_{\text {hom }} \subset \mathrm{CH}^{1}(X)$ the group of cycles homologically equivalent to zero as the kernel of the composition

$$
\mathrm{CH}^{1}(X) \stackrel{\sim}{\longrightarrow} \mathrm{CH}^{1}(U) \rightarrow H_{\mathrm{ett}}^{2}\left(U, \mathbb{Q}_{\ell}(1)\right), \quad \ell \neq \text { char } k .
$$

If $X$ is just normal and proper, we can use Chow's lemma [8, Theorem 5.6.1] to find a projective, normal modification $\pi: \widetilde{X} \rightarrow X$-where $\pi$ is a projective, surjective, birational morphism, and $\widetilde{X}$ is a normal, projective variety (we can assume normality because normalization is a projective morphism). For a normal, projective variety $Y$, define the group $B^{1}(Y)=\left(\mathrm{CH}^{1}(Y) / \mathrm{CH}^{1}(Y)_{\text {hom }}\right) \otimes \mathbb{Q}$.

Note that if $X$ is smooth, then algebraic equivalence (see $[6,10.3]$ ) and numerical equivalence tensored with $\mathbb{Q}$ coincide for codimension-one cycles $([9,6.3])$, also numerical and homological equivalences tensored with $\mathbb{Q}$ coincide for codimensionone cycles $[1,3.4 .6 .1]$. In particular, $B^{1}(Y)=\mathrm{NS}(Y) \otimes \mathbb{Q}$.

Given a morphism $f: Y \rightarrow X$, we have a few different notions of pullback of divisors. If $f$ is birational, we let $f^{-1}: Z^{1}(Y) \rightarrow Z^{1}(X)$ denote the strict transform; in general, this does not descend to a morphism on $\mathrm{CH}^{1}$. If $X$ is normal and projective, and $f$ is the inclusion of a linear section, we may define the intersection map $f^{*}: \mathrm{CH}^{1}(X) \rightarrow \mathrm{CH}^{1}(Y)$, which is proven to be well-defined in [6, Proposition 2.6]. If $f$ is flat, $f^{*}: \mathrm{CH}^{1}(X) \rightarrow \mathrm{CH}^{1}(Y)$ denotes the flat pullback. In Proposition 2.3 and Corollary 2.4 we will use these to construct pullback morphisms for resolutions of singularities of surfaces, and resolutions of singularities of generic 2-dimensional linear sections, respectively.

Lemma 2.1 Let $X$ be a normal projective integral variety defined over an algebraically closed field $k$. Then:

(i) the algebraic and homological equivalences tensored over $\mathbb{Q}$ coincide for codimension-one cycles on $X$;

(ii) $\operatorname{dim}_{\mathbb{Q}} B^{1}(X)<\infty$, and this number is independent of $\ell$ chosen in the definition.

Proof Let $U \subseteq X$ be the smooth locus. Note that if a codimension-one cycle $\alpha \in$ $Z^{1}(U)$ is algebraically equivalent to 0 , then it is homologically equivalent to 0 . Hence, for (i), it is enough to show that if the class of $\alpha$ is 0 , then $\alpha$ is algebraically equivalent to zero. Let $f: \widetilde{X} \rightarrow X$ be a smooth projective alteration of degree $d$, such that for $Z=X \backslash U, \widetilde{Z}=f^{-1}(Z)$ is a simple normal crossings divisor [10]. Let $\widetilde{U}$ be the inverse image of $U$ and $U_{0} \subset U$ an open subset, such that the induced morphism $\widetilde{U}_{0}=f^{-1}\left(U_{0}\right) \rightarrow U_{0}$ is finite of degree $d$. Note that one can assume that the complement of $U_{0}$ in $U$ is of codimension at least two: indeed, this follows from the fact that in the Stein factorisation $\widetilde{X} \rightarrow Y \rightarrow X$ of $f$, with $\widetilde{X} \rightarrow Y$ birational and $Y \rightarrow X$ finite of degree $d$, the map $\widetilde{X} \rightarrow Y$ is an isomorphism over any point of codimension-one, since $X$, and therefore also $Y$, is normal.

Assume now that $\alpha$ is homologically equivalent to 0 on $X$. Since $\widetilde{Z}$ is a simple normal crossings divisor, we deduce that there exists a codimension-one cycle $\beta$ supported on $\widetilde{Z}$, so that $f^{-1}(\alpha)+\beta$ is homologically equivalent to 0 on $\widetilde{X}$. Since $X$ is smooth, the discussion above the lemma shows that there is an integer $n$ such that $n\left(f^{-1}(\alpha)+\beta\right)$ is algebraically equivalent to 0 on $\widetilde{X}$. Hence, its restriction $n f^{-1}(\alpha)$ 
to $\widetilde{U}_{0}$ is algebraically equivalent to 0 . Since $\widetilde{U}_{0} \rightarrow U_{0}$ is finite of degree $d$, we deduce that $n \alpha$ is algebraically equivalent to 0 on $U_{0}$, hence on $U$, as the complement of $U_{0}$ in $U$ is of codimension at least two, and we obtain (i).

For (ii), the independence of $\ell$ follows from (i). For the finiteness, it is enough to show that there is no infinite collection of divisors in $X$ with $\mathbb{Q}$-linearly independent classes in $H_{\text {ét }}^{2}\left(U_{0}, \mathbb{Q}_{\ell}(1)\right)$. Via the trace map $H_{\text {ét }}^{2}\left(\widetilde{U}_{0}, \mathbb{Q}_{\ell}(1)\right) \rightarrow H_{\text {ét }}^{2}\left(U_{0}, \mathbb{Q}_{\ell}(1)\right)$ (see [5, Exposé IX, (5.1.4)]) it is enough to establish the same property for $\widetilde{U}_{0}$, which follows from the fact that the Néron-Severi group of the smooth variety $\widetilde{X}$ is finitely generated.

The lemma above allows us to make the following definition, independently of $\ell$.

Definition 2.2 The Weil divisor rank $\rho_{\mathrm{W}}(X)$ of $X$ is the minimum dimension of the $\mathbb{Q}$-vector space $B^{1}(\widetilde{X})$ over all projective, normal modifications $\pi: \widetilde{X} \rightarrow X$ of $X$.

In what follows we will fix $\pi: \widetilde{X} \rightarrow X$ a projective, normal modification for which the dimension of the group $B^{1}(\tilde{X})$ is minimized.

Proposition 2.3 Let $T^{\prime}$ be a normal, projective surface, $\beta: T \rightarrow T^{\prime}$ a resolution of singularities which is an isomorphism on the smooth locus of $T^{\prime}, \Gamma \subseteq B^{1}(T)$ the $\mathbb{Q}$-vector space generated by the divisors contracted by $\beta$, and $\Gamma^{\perp}$ the orthogonal complement of $\Gamma$ in $B^{1}(T)$ under the intersection pairing. Then $\beta^{-1}: Z^{1}\left(T^{\prime}\right) \rightarrow Z^{1}(T)$ induces an isomorphism $\beta^{-1}: B^{1}\left(T^{\prime}\right) \rightarrow B^{1}(T) / \Gamma$, and composing with the projection to the orthogonal complement of $\Gamma$ gives a canonical morphism $\beta^{*}: B^{1}\left(T^{\prime}\right) \rightarrow$ $\Gamma^{\perp} \subseteq B^{1}(T)$.

Proof Let $\eta: V \rightarrow T$ be the inclusion of the inverse image in $T$ of the smooth locus of $T^{\prime}$. Each exceptional divisor is in the kernel of the flat pullback morphism $\eta^{*}: \mathrm{CH}^{1}(T) \rightarrow \mathrm{CH}^{1}(V)$. The image of $\Gamma \otimes \mathbb{Q}_{\ell}$ under the cycle map in $H_{\text {ét }}^{2}\left(T, \mathbb{Q}_{\ell}(1)\right)$ is exactly the kernel of the restriction morphism to $H_{\text {ét }}^{2}\left(V, \mathbb{Q}_{\ell}(1)\right)$ by inductive use of the Gysin sequence [11, Corollary 16.2]. But every element of $Z^{1}(T)$ can be written as the strict transform of an element of $Z^{1}\left(T^{\prime}\right)$ plus a linear combination of exceptional divisors. Therefore, $\beta^{-1}: B^{1}\left(T^{\prime}\right) \rightarrow B^{1}(T) / \Gamma$ is an isomorphism. As the intersection pairing on $\Gamma$ is negative-definite by [2, Remark after Theorem 6.12], the Hodge index theorem gives that $B^{1}(T)=\Gamma \oplus \Gamma^{\perp}$, so $\Gamma^{\perp} \simeq B^{1}(T) / \Gamma$, and we have defined $\beta^{*}$.

As an immediate corollary, we have

Corollary 2.4 Let $X$ be normal and projective. Let $\iota: T^{\prime} \rightarrow X$ be an intersection of hyperplane sections of some projective embedding of $X$, smooth on the intersection with the smooth locus of $X$ (such sections are generic by [14, Theorem 1]), of dimension two. Let $\beta: T \rightarrow T^{\prime}$ be a resolution of singularities which is an isomorphism on the smooth locus of $T^{\prime}$ and for which the exceptional divisors are simple normal crossings and let $\phi: T \rightarrow X$ be the composition $\iota \beta$. Then we have a pullback homomorphism $p_{T}=\beta^{*} \circ \iota^{*}: B^{1}(X) \rightarrow B^{1}(T)$.

We now recall some facts about Albanese varieties. Let $Y$ be a variety defined over an algebraically closed field $k, Y_{i}$ its irreducible components, and let $x_{0, i}$ be a smooth 
point of each $Y_{i}$. We say a rational map (resp. morphism) $f: Y \rightarrow A$ with $A$ an abelian variety is admissible if $f$ is defined at each $x_{0, i}$ and $f\left(x_{0, i}\right)=0$. Following Ghorpade and Lachaud [7, Section 9], we call an Albanese-Weil variety $\operatorname{Alb}_{\mathrm{w}}(Y)$ (resp., an Albanese-Serre variety $\operatorname{Alb}_{\mathrm{s}}(Y)$ ) of $Y$ an abelian variety $A$ over $k$ with an admissible rational map $f$ (resp., morphism) from $Y$ to $A$, such that the following universal property holds: for any admissible rational map $g$ (resp., morphism) from $Y$ to an abelian variety $B$ there is a homomorphism of abelian varieties $\widetilde{g}: A \rightarrow B$ such that $g=\widetilde{g} \circ f$. We have the following properties:

- The variety $\operatorname{Alb}_{\mathrm{W}}(Y)$ and the universal rational map $Y \rightarrow \operatorname{Alb}_{\mathrm{W}}(Y)$ exist, are independent of the choice of $x_{0, i}$ up to a translation, and $\operatorname{Alb}_{\mathrm{w}}(Y)=\prod_{i} \operatorname{Alb}_{\mathrm{w}}\left(Y_{i}\right)$.

- If $Y$ is normal, the variety $\operatorname{Alb}_{\mathrm{s}}(Y)$ exists, and is dual to the reduced Picard variety $\left(\mathrm{Pic}_{Y / k}^{0}\right)_{\text {red }}$ [7, paragraph after Example 9.2].

- If $Y$ is smooth, the variety $\operatorname{Alb}_{\mathrm{s}}(Y)$ coincides with $\operatorname{Alb}_{\mathrm{w}}(Y)$ and for $Y$ normal, there is a canonical surjective map $v: \operatorname{Alb}_{\mathrm{W}}(Y) \rightarrow \operatorname{Alb}_{\mathrm{s}}(Y)$ with connected kernel [7, Proposition 9.1].

- A birational morphism $Y \rightarrow X$ of varieties induces an isomorphism $\operatorname{Alb}_{\mathrm{w}}(Y) \rightarrow$ $\mathrm{Alb}_{\mathrm{w}}(X)$ (this follows straight from the definition), so a resolution of singularities $Y \rightarrow X$ induces an isomorphism $\operatorname{Alb}_{\mathrm{s}}(Y) \rightarrow \operatorname{Alb}_{\mathrm{w}}(X)$.

We need the following Lefschetz-type property [7, Proposition 9.4].

Proposition 2.5 Let $X \hookrightarrow \mathbb{P}^{N}$ be an embedding of $X$ into a projective space. If $i: Y \hookrightarrow X$ is a general linear section of $X$ of dimension $d \geqslant 2$, the canonical map $i_{*}: \operatorname{Alb}_{\mathrm{w}}(Y) \rightarrow \operatorname{Alb}_{\mathrm{w}}(X)$ induced by $i$ is a purely inseparable isogeny.

\subsection{The torsion case}

We start with the following easy lemma.

Lemma 2.6 Let $\Delta_{1}$ and $\Delta_{2}$ be two effective, disjoint divisors on a proper, normal variety $Y$ over a field $k$, and suppose that

$$
\Delta_{1}-\Delta_{2} \sim_{\text {lin }} 0 .
$$

Then there exists a morphism $f: Y \rightarrow \mathbb{P}_{k}^{1}$ such that $\Delta_{1}=f^{-1}(0)$ and $\Delta_{2}=f^{-1}(\infty)$.

Proof By assumption, there exists a rational function $g$ on $Y$ such that $\Delta_{1}-\Delta_{2}=$ $\operatorname{div}(g)$. Then we define a map $f: Y \rightarrow \mathbb{P}_{k}^{1}$ by $f(x)=[g(x): 1]$ if $x$ is not in the support of $\Delta_{2}$ and [1:0] (equivalently, [1:g(x)] if $x$ is not in the support of $\Delta_{1}$ ) otherwise. Since the divisors $\Delta_{1}$ and $\Delta_{2}$ are disjoint, we get a well-defined map as required.

Let now $X$ be as in Theorem 1.1. We assume first that $X$ is projective. Since $\# I \geqslant \rho_{\mathrm{w}}(X)+1$, there is a subset $J \subseteq I$ and a nontrivial linear combination $D=$ $\sum_{j \in J} \lambda_{j} D_{j} \in Z^{1}(X)$, where $\lambda_{j} \in \mathbb{Z}$ and $D \in \mathrm{CH}_{\text {hom }}^{1}(X)$.

Proposition 2.7 If there exists $N>0$ such that $N D \sim_{\operatorname{lin}} 0$, then there is a surjective morphism $f: X \rightarrow \mathbb{P}_{k}^{1}$ such that for any $j \in J$ the divisor $D_{j}$ is contained in a fiber of $f$. 
Proof It suffices to write $N D=\Delta_{1}-\Delta_{2}$ as a difference of two effective (and disjoint) divisors and apply Lemma 2.6. We obtain a map $f: X \rightarrow \mathbb{P}_{k}^{1}$ satisfying the required properties. Note that for each $i \in I \backslash J$ the divisor $D_{i}$ is a subset of a fiber of $f$ : otherwise, for each $j \in J$, there would exist some $i \in I$ so that $D_{j}$ would intersect $D_{i}$, contradicting disjointness.

To handle the non-torsion case, we need that non-torsion elements of $\mathrm{CH}^{1}$ specialize under generic hyperplane sections to non-torsion elements.

Proposition 2.8 Let $X \hookrightarrow \mathbb{P}^{N}$ be an embedding of $X$ into a projective space. If $D$ is a non-torsion element of $\mathrm{CH}^{1}(X)$ then for a general linear section $\tau: Y \subset X$ of dimension $d \geqslant 2$ the restriction $\tau^{*} D$ given by intersection of $D$ with $Y$ to $\mathrm{CH}^{1}(Y)$ is also a non-torsion element.

Proof By induction, it suffices to prove the theorem for general $Y$ of codimensionone. We may assume $Y$ is normal [14, Theorem 7], and that $Y$ contains no irreducible component of $D$, as $Y$ is basepoint-free. Suppose there is an integer $N_{Y}$ and a function $f_{Y} \in k(Y)$ such that $N_{Y} \tau^{*} D=\operatorname{div}\left(f_{Y}\right)$ in $Z^{1}(Y)$. We can lift the function $f_{Y}$ to an element $F_{Y} \in \mathcal{O}_{X, Y} \subset k(X)$. Define $D^{\prime}=N_{Y} D-\operatorname{div}\left(F_{Y}\right)=\sum a_{i} Z_{i}$ with $a_{i} \in \mathbb{Z}$ and $Z_{i}$ irreducible components of $D^{\prime}$, that are included in $X \backslash Y$ by construction. Since $Y$ is ample, $Y$ intersects every proper codimension-one subvariety, so $X$ has no proper codimension-one subvariety contained in $X \backslash Y$, so that we have $\operatorname{div}\left(F_{Y}\right)-N_{Y} D=0$ in $\mathrm{CH}^{1}(X)$, contradicting our assumption on $D$.

\subsection{The Hodge index theorem and the general case}

Let $\widetilde{S}$ be a projective resolution of singularities of a generic, normal, linear surface section $S \subseteq X$, whose smooth locus is exactly the intersection of the smooth locus of $X$ with $S$. As a simple linear-algebraic corollary of the Hodge index theorem, we have

Proposition 2.9 Let $H=\left\{H_{j}\right\}_{j \in J} \subseteq B^{1}(\widetilde{S})$ be an orthogonal subset of nonzero elements-that is, $H_{j} \cdot H_{j^{\prime}}=0$ for each $j \neq j^{\prime}$. Assume furthermore that $H$ is contained in a subspace $V \subseteq B^{1}(\widetilde{S})$ of dimension d for which there exists $M \in V$ so that $M \cdot M>0$. Define

$$
J_{+}\left(\text {resp., } J_{-}, J_{0}\right)=\left\{j \in J: H_{j} \cdot H_{j}>(\text { resp., }<,=) 0\right\} .
$$

Then:

(i) $J_{+} \cup J_{-}$is a linearly independent set, and \# $J_{+} \leqslant 1$ and \# $J_{-} \leqslant d-1$.

(ii) If \# $\left(J_{+} \cup J_{0}\right) \geqslant 2$ then $\# J_{-} \leqslant d-2$, \# $J_{+}=0$ and \# $J_{0} \geqslant \# J-(d-2)$.

(iii) The span of $J_{0}$ is at most one-dimensional.

The pullback $\left\{\widetilde{D}_{i}\right\}_{i \in I}$ of $\left\{D_{i}\right\}_{i \in I}$ to $B^{1}(\widetilde{S})$ is an orthogonal set of nonzero elements, and is contained in the image of $B^{1}(X)$, by Proposition 2.4. The restriction of a general ample divisor to $\widetilde{S}$ is likewise ample, so the image of $B^{1}(X)$ in $B^{1}(\widetilde{S})$ is a subspace of dimension $\leqslant \rho_{\mathrm{w}}(X)$ which contains an element of positive self-intersection. By 
Proposition 2.9, \# $\left(J_{+} \cup J_{-}\right) \leqslant \rho_{\mathrm{W}}(X)-2$, so in fact \# $J_{0} \geqslant 3$. Let $i, j, t$ be distinct elements of $J_{0}$, and let $F=a \widetilde{D}_{i}-b \widetilde{D}_{j}$ be an integral linear combination in $\mathrm{CH}^{1}(\widetilde{S})_{\text {hom }}$. Let $I^{\prime}=I \backslash\{i, j\}$.

Proposition 2.10 If $F$ is not torsion, then for each $l \in I^{\prime}$, the map $\operatorname{Alb}_{\mathrm{W}}\left(D_{l}\right) \rightarrow$ $\mathrm{Alb}_{\mathrm{w}}(X)$ is not surjective.

Proof Fix a projective embedding $X \subset \mathbb{P}^{N}$. By Proposition 2.5, if $\tau: S \subset X$ is a general linear section of $X$ of dimension two, then $S$ is normal, and we have an isogeny $\operatorname{Alb}_{\mathrm{w}}(S) \rightarrow \mathrm{Alb}_{\mathrm{w}}(X)$. By Proposition 2.8, the restriction of $F^{\prime}$ of $F$ to $\mathrm{CH}^{1}(S)_{\text {hom }}$ is not a torsion element. Let $v: \widetilde{S} \rightarrow S$ be a resolution of singularities. Since $S$ is normal, we may assume that $v$ is an isomorphism over the smooth locus $S^{\mathrm{sm}}$, which contains all codimension-one points of $S$. Let $\widetilde{D}_{l}$ be a union of normalizations of the components of $v^{-1}\left(D_{l} \cap S\right)$ (that is, the inverse image of the intersection of $D_{l}$ intersected with $S$ ). Since the support of $F$ is disjoint from $D_{l}$, the line bundle defined by $F$ becomes trivial on $\widetilde{D}_{l}$ and we conclude that $\widetilde{D}$ is a non-torsion element in the kernel of the map $\operatorname{Pic}^{0}(\widetilde{S}) \rightarrow \operatorname{Pic}^{0}\left(\widetilde{D}_{l}\right)$. By duality, we obtain that the map $\operatorname{Alb}_{\mathrm{s}}\left(\widetilde{D}_{l}\right) \rightarrow \operatorname{Alb}_{\mathrm{s}}(\widetilde{S})$ is not surjective. The maps $\operatorname{Alb}_{\mathrm{s}}(\widetilde{S}) \rightarrow \operatorname{Alb}_{\mathrm{W}}(S) \rightarrow \operatorname{Alb}_{\mathrm{w}}(X)$ are isogenies, so $\operatorname{Alb}_{\mathrm{w}}\left(\widetilde{D}_{l}\right) \rightarrow \operatorname{Alb}_{\mathrm{w}}(\widetilde{X})$ cannot be surjective.

Proof of Theorem 1.1 In Proposition 2.7, we established the result if $F$ is a torsion element in $\mathrm{CH}^{1}(X)$. Consider now the general case. Assume $F \in \mathrm{CH}^{1}(X)$ nontorsion. Let $i, j, t$ be distinct elements of $J_{0}$ as before. By Proposition 2.10, the map $\operatorname{Alb}_{\mathrm{W}}\left(\widetilde{D_{t}}\right) \rightarrow \operatorname{Alb}_{\mathrm{W}}(X)$ is not surjective.

By the universal property of the Albanese variety, we have the following commutative diagram:

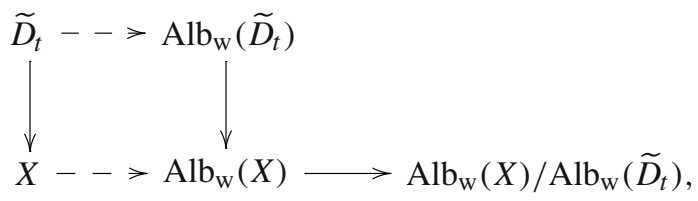

showing that $\widetilde{D}_{t}$ is contracted by the composite rational map $g: X \rightarrow \operatorname{Alb}_{\mathrm{w}}(X) /$ $\operatorname{Alb}_{\mathrm{W}}\left(\widetilde{D}_{t}\right)$. Let us show that the image of $g$ is a curve:

- We have $\operatorname{dim} \operatorname{Im}(g)>0$ since the image of $X$ in $\operatorname{Alb}_{\mathrm{W}}(X)$ generates the abelian variety $\operatorname{Alb}_{\mathrm{w}}(X)$ by the universal property and $\operatorname{Alb}_{\mathrm{w}}\left(D_{t}\right) \rightarrow \operatorname{Alb}_{\mathrm{w}}(X)$ is not surjective.

- If $\operatorname{dim} \operatorname{Im}(g)$ were greater than one, the image of the dimension of $g$ restricted to $\widetilde{S}$ would also have image of dimension two. The morphism $g$ restricted to $\widetilde{S}$ is a regular map, because it factors through $\operatorname{Alb}_{\mathrm{s}}(\widetilde{S}) / \operatorname{Alb}_{\mathrm{w}}\left(\widetilde{D}_{t}\right)$, and the map from $\widetilde{S} \rightarrow \operatorname{Alb}_{\mathrm{s}}(\widetilde{S})$ is defined everywhere. Any effective divisor on $\widetilde{S}$ contracted by $g$ would need to have negative self-intersection [2, Remark after Theorem 6.12]. But $D_{t}$ is contracted and has self-intersection 0 , so the image has to be of dimension $<2$.

We then see immediately that $\widetilde{D}_{j}$ is also contracted and should be (a multiple of) a fiber of $g$ restricted to $\widetilde{S}$, since its self-intersection is zero. Therefore, $D_{j}$ and $D_{t}$ are 
(multiples of) fibers of the rational map to $\operatorname{Alb}_{\mathrm{W}}(X) / \operatorname{Alb}_{\mathrm{w}}\left(D_{t}\right)$; as they are disjoint, and $X$ is normal, $g$ is in fact a regular map-that is, it is defined everywhere.

Therefore, the image of $g: X \rightarrow \operatorname{Alb}_{\mathrm{w}}(X) / \operatorname{Alb}_{\mathrm{w}}\left(D_{t}\right)$ is a curve $C^{\prime}$, and all divisors $D_{l}$ for $l \in I^{\prime}$ are contained in fibers. If now

$$
X \stackrel{f}{\rightarrow} C \rightarrow C^{\prime}
$$

is the Stein factorization of $g, C$ is a normal curve, in which the $D_{l}$ are contained in fibers for $l \in I^{\prime}$. By [3, Fact $\left.1.1 \mathrm{~d}\right], D_{t}$ is in fact a multiple of an entire fiber of $f$; as $D_{i}$ and $D_{j}$ are disjoint from $D_{t}$, both $D_{i}$ and $D_{j}$ are contained in fibers, and $D_{\sigma}$ which are (multiples of) fibers of $f$ are exactly those for which $\sigma \in J_{0}$. We may thus set $J$ to be $J_{0}$.

Finally, if $X$ is normal and proper, consider $\left\{\pi^{-1}\left(D_{i}\right)\right\}_{i \in I}$, where $\pi: \widetilde{X} \rightarrow X$ is a birational morphism given by the Chow lemma, with $\widetilde{X}$ projective. By Zariski's main theorem, each $\pi^{-1}\left(D_{i}\right)$ is connected. Then there exists a function $\widetilde{f}: \widetilde{X} \rightarrow C$ for which each of $\pi^{-1}\left(D_{i}\right)$ is contained in a fiber, and for at least two (in fact, three) $m_{1}, m_{2}, m_{3} \in I, \pi^{-1}\left(D_{m_{i}}\right)$ is a (multiple of) a fiber. As $X$ is normal, by Zariski's main theorem, to check that $\tilde{f}$ factors through a function to $X$ we must merely check that none of the divisors that $\pi$ contracts intersects $\pi^{-1}\left(D_{m_{1}}\right)$ and $\pi^{-1}\left(D_{m_{2}}\right)$. But if a divisor that $\pi$ contracts intersected both of them, $D_{m_{i}}$ would not be disjoint.

\section{Disjoint divisors on affine varieties}

In this section we prove Theorems 1.2 and 1.3. Let $y, z$ be the coordinates of $\mathbb{A}_{k}^{2}$. Consider the following family, constructed recursively:

1. Define $d_{0}=1, f_{0}(y, z)=z^{d_{0}}$, and $D_{0}=V\left(f_{0}\right)$, the zero locus of $f_{0}$.

2. Define $d_{1}=2, f_{1}(y, z)=y z^{d_{1}}+1$, and $D_{1}=V\left(f_{1}\right)$.

3. Let $P_{2} \in \mathbb{A}_{k}^{2} \backslash\left(D_{0} \cup D_{1}\right)$ and define

$$
a_{2}=-f_{1}(P) / f_{0}(P)^{2 d_{1}-1}
$$

Since $P_{2} \notin D_{1}, a_{2} \neq 0$. Define $d_{2}=2 d_{1}$,

$$
f_{2}(y, z)=a_{2} f_{0}(y, z)^{d_{2}-1}+f_{1}(y, z)^{d_{1}},
$$

and $D_{2}=V\left(f_{2}\right)$. Note that in each case, $d_{i}=\operatorname{deg}_{z} f_{i}$, the degree of $f_{i}$ as a polynomial in $z$.

4. Let now $n>2$, we give a recursive definition of $f_{n}$, given $f_{i}$ when $i<n$. We define $D_{i}=V\left(f_{i}\right)$ and $d_{i}=\operatorname{deg}_{z} f_{i}$. Define now $d_{n}=\sum_{i=1}^{n-1} d_{i}$. Let $P_{n} \in \mathbb{A}_{k}^{2} \backslash \bigcup_{i=0}^{n-1} D_{i}$, define

$$
a_{n}=-\prod_{i=1}^{n-1} f_{i}(P) / f_{0}(P)^{d_{n}-1}
$$


Again, $a_{n} \neq 0$. Define

$$
f_{n}(y, z)=a_{n} f_{0}(y, z)^{d_{n}-1}+\prod_{i=1}^{n-1} f_{i}(y, z)
$$

and $D_{n}=V\left(f_{n}\right)$, by construction, $d_{n}=\operatorname{deg}_{z} f$.

If we enumerate the $k$-points of $\mathbb{A}_{k}^{2}$, we may choose our $P_{i}$ so that $\bigcup_{i} D_{i}(k)=$ $\mathbb{A}^{2}(k)$. By construction, $D_{i}$ are pairwise-disjoint: the radical of the ideal generated by $f_{n}$ and $f_{i}$ for $0<i<n$ contains $f_{0}$ and $f_{i}$; and by construction, $f_{i}$ and $f_{0}$ have no common zeroes.

We now prove that each $f_{n}$ is irreducible, and that no infinite subset of $D_{i}$ could be contained in the fibers of a non-constant morphism.

To prove that $f_{n}$ is irreducible, we will change coordinates. We view $\mathbb{P}_{k}^{1}=\mathbb{A}_{k}^{1} \cup\{\infty\}$. Let $\bar{X}=\mathbb{P}_{k}^{1} \times \mathbb{P}_{k}^{1}$, and use the coordinates $y, z$ to embed $\mathbb{A}_{k}^{2}$ as an open subset

$$
\mathbb{A}_{k}^{2} \stackrel{\sim}{\longrightarrow} \mathbb{A}_{k}^{1} \times \mathbb{A}_{k}^{1} \hookrightarrow \bar{X} ;
$$

call this open subset $X_{1}$. Let $\overline{D_{i}}$ be the Zariski closure of $D_{i}$ in $\bar{X}$. Define $X_{2} \subseteq \bar{X}$ as $\mathbb{A}_{k}^{1} \times\left(\mathbb{P}_{k}^{1} \backslash\{0\}\right)$; this is isomorphic to $\mathbb{A}^{2}$ with coordinates $y, x=1 / z$. Let $D_{i}^{\prime}=\overline{D_{i}} \cap X_{2}$. The defining ideals for $D_{i}^{\prime}$ are generated by:

- $f_{1}^{\prime}(y, x)=y+x^{2}$

- $f_{2}^{\prime}(y, x)=a_{2} x+\left(y+x^{2}\right)^{2}$;

- $f_{n}^{\prime}(y, x)=a_{n} x+\prod_{i=1}^{n-1} f_{i}(y, x)$.

By Eisenstein's criterion, applied to the ring $k(x)[y]$, all polynomials $f_{n}^{\prime}$ are irreducible. Therefore, to check whether $f_{i}$ are irreducible, we must merely check that $V\left(f_{i}\right)$ does not have any component contained in $X_{1} \backslash\left(X_{1} \cap X_{2}\right)$. But $X_{1} \backslash\left(X_{1} \cap X_{2}\right)$ is just $D_{0}$, and $D_{i} \cap D_{0}=\varnothing$ for $i>0$.

As polynomials $f_{i}$ have unbounded degree and are irreducible, their zero sets could not be the fibers of a morphism (or even a rational map!) to a curve and Theorem 1.2 is proved.

To prove Theorem 1.3, we choose algebraically independent $y, z$ in the ring of regular functions on $X$ for which $V(y)$ and $V(z)$ are irreducible, and construct $f_{i}$ and $D_{i}$ as above (replacing $D_{i}$ with its finite set of connected components at each stage, if necessary). Any function to a curve with $D_{i}$ as fibers would factor through the map to $\mathbb{A}^{2}$ given by $y$ and $z$.

Remark 3.1 This procedure is by no means unique. For instance, one could replace $f_{0}$ and $f_{1}$ by any other two irreducible polynomials with no common roots in $\mathbb{A}_{k}^{2}$, and the above construction works. However, the following questions remain open:

- Does there exist an example as above where the curves are all smooth?

- In any example as above, is the geometric genus of the curves necessarily unbounded? (That is, could we find such a counterexample consisting of only rational curves?) 
- In any example as above, does there necessarily exist a divisor $D$ such that $\#\left(D \cap D_{i}\right)$, the set-theoretic intersection, is unbounded?

We now prove Theorem 1.4, that any uncountable set of disjoint divisors must form a family. In this proof, $X$ will be normal and affine; the theorem follows immediately for all normal varieties from this case.

Proposition 3.2 Let $X$ be a normal quasi-projective variety over an uncountable algebraically closed field $k$. Let $\left\{D_{i}\right\}_{i \in I}$ be an uncountable collection of pairwisedisjoint, reduced, connected, codimension-one, closed subvarieties of $X$. Then there exist a smooth projective curve $C$ defined over $k$ and a dominant morphism $\varphi: X \rightarrow C$ so that for any $i \in I$ the divisor $D_{i}$ is contained in a fiber of $f$.

Proof Let $X \subset \bar{X}$ be a projective model of $X$. We may assume that $\bar{X}$ is normal. Let $\bar{D}_{i}$ be the closure of $D_{i}$ in $\bar{X}$. Note that if there is a subset $I_{0} \subset I$ with $\# I_{0} \geqslant \rho_{\mathrm{W}}(\bar{X})+1$, such that $\bar{D}_{i}, i \in I_{0}$, are disjoint, then we can apply Theorem 1.1 for $\bar{X}$ to get a map $g: \bar{X} \rightarrow C$ such that all $\bar{D}_{i}, i \in I_{0}$, are contained in the fibers of $g$. Since for $i \in I$, $D_{i}$ are disjoint, we can take $\varphi$ to be the restriction of $g$ to $X$.

There is an alteration $f: \bar{Y} \rightarrow \bar{X}$, such that $\bar{Y}$ is smooth and for $Y=f^{-1}(X)$, we have $Y_{\infty}=\bar{Y} \backslash Y$ is a simple normal crossings divisor [10]. Let $F_{i}=f^{-1}\left(D_{i}\right)$, then $F_{i}$ are disjoint and cover $Y$. Let $\bar{F}_{i} \subset \bar{Y}$ be the closure of $F_{i}$ in $\bar{Y}$. Since $\bar{Y}$ is smooth, each $\bar{F}_{i}$ gives a class in the Picard group $\operatorname{Pic}(\bar{Y})$. Since $\mathrm{NS}(\bar{Y})$ is countable, we obtain that there is an uncountable $J \subset I$ for which the divisors $\bar{F}_{j}, j \in J$, all have the same class $\alpha \in \mathrm{NS}(\bar{Y})$.

Lemma 3.3 There is an infinite subset $J^{\prime} \subset J$, a finite set of irreducible divisors $\left(E_{t}\right)_{t \in T} \subset Y_{\infty}$ and $M>0$ such that for any $i, j \in J^{\prime}$ one has $\bar{F}_{i} \cdot \bar{F}_{j}=\sum_{t \in T} a_{t} E_{t}$ with $0 \leqslant a_{t} \leqslant M$.

Proof Since the divisors $\left(\bar{F}_{j}\right)_{j \in J}$ intersect only on $Y_{\infty}$, for a fixed $j \in J$, any intersection $\bar{F}_{j} \cap \bar{F}_{j^{\prime}}$ with $j^{\prime} \in J$ is supported on components of $\bar{F}_{j} \cap Y_{\infty}$, and the intersections $\bar{F}_{j} \cap \bar{F}_{j^{\prime}}$ are all in the same Néron-Severi class $\alpha^{2}$ on $Y_{\infty}$.

Let $j_{0} \in J$. Since $J$ is infinite, there is an infinite subset $J_{1} \subset J$ such that for any $j, j^{\prime} \in J_{1}$ one has $H_{0}=\bar{F}_{j_{0}} \cdot \bar{F}_{j}=\bar{F}_{j_{0}} \cdot \bar{F}_{j^{\prime}}$ as a divisor (not only a class) on $Y_{\infty}$; we may also assume that this intersection is nonzero. Fix now $j_{1} \in J_{1}$. Similarly, one finds an infinite subset $J_{2} \subset J_{1}$ such that for any $j, j^{\prime} \in J_{2}$ one has $H_{1}=\bar{F}_{j_{1}} \cdot \bar{F}_{j}=\bar{F}_{j_{1}} \cdot \bar{F}_{j^{\prime}}$.

By the same procedure, we construct inductively the sets $J_{n}$ and the divisors $H_{n}$. Since all divisors $\bar{F}_{j}$ have the same divisor class, after a finite number of steps we obtain $H_{n+r} \subset \bigcup_{m<n} H_{m}$ for all $r \geqslant 0$. We may then take $J^{\prime}$ to be that $J_{n}$ and $T$ the set of irreducible components of $\bigcup_{m<n} H_{m}$.

The lemma above gives the following bound on $\bar{X}$ : there is an integer $N$ such that for any closed $Z \subset \bar{X}$ that is a (set-theoretic) component of the intersection of $\bar{D}_{j}$ and $\bar{D}_{j^{\prime}}, j \in J^{\prime}$, we have that locally in $\mathcal{O}_{X, Z}$, the ideal of the intersection of $\bar{D}_{j}$ and $\bar{D}_{j^{\prime}}$ is contained in at most the $N^{\text {th }}$ power of the maximal ideal $\mathfrak{m}_{Z}^{N} \subset \mathcal{O}_{X, Z}$ of $Z$. Hence after a finite number of blow-ups $\widetilde{X} \rightarrow \bar{X}$ centered at $X_{\infty}$, the strict transforms $\widetilde{D}_{j}$ of $\bar{D}_{j}$ do not intersect. Now we can apply Theorem 1.1 to $\widetilde{X}$ and the family $\left(\widetilde{D}_{j}\right)_{j \in J^{\prime}}$ to get a map $f: \widetilde{X} \rightarrow C$, such that the restriction $\phi$ of $f$ to $X$ satisfies the conclusion of the theorem. 
Acknowledgments The third author thanks Madhav Nori, Keerthi Madapusi-Pera, Mihnea Popa, and Jesse Kass for helpful conversations. We gratefully acknowledge Burt Totaro and John Christian Ottem for pointing us in the direction of [15] and [12], after this paper was first posted on the arXiv.

\section{References}

1. André, Y.: Une Introduction aux Motifs (Motifs Purs, Motifs Mixtes, Périodes). Panoramas et Synthèses, vol. 17. Société Mathématique de France, Paris (2004)

2. Artin, M.: Algebraization of formal moduli. II. Existence of modifications. Ann. Math. 91(1), 88-135 (1970)

3. Artin, M., Winters, G.: Degenerate fibres and stable reduction of curves. Topology 10(4), 373-383 (1971)

4. Deligne, P.: Cohomologie Étale. In: Séminaire de Géométrie Algébrique du Bois-Marie SGA 4 1/2. Lecture Notes in Mathematics, vol. 569. Springer, Berlin (1977)

5. Dold, A., Eckmann, B. (eds.): Théorie des Topos et Cohomologie Étale des Schémas. Tome 3 (SGA4). Lecture Notes in Mathematics, vol. 305. Springer, Berlin (1973)

6. Fulton, W.: Intersection Theory. Ergebnisse der Mathematik und ihrer Grenzgebiete, vol. 2. Springer, Berlin (1984)

7. Ghorpade, S.R., Lachaud, G.: Étale cohomology, Lefschetz theorems and number of points of singular varieties over finite fields. Mosc. Math. J. 2(3), 589-631 (2002)

8. Grothendieck, A.: Éléments de géométrie algébrique. II. Étude globale élémentaire de quelques classes de morphismes. Inst. Hautes Études Sci. Publ. Math. 8, 5-205 (1961)

9. Kleiman, S.L.: The Picard scheme. In: Fantechi, B., et al. (eds.) Fundamental Algebraic Geometry. Mathematical Surveys and Monographs, vol. 123, pp. 235-300. American Mathematical Society, Providence (2005)

10. Illusie, L.: On Gabber's refined uniformization (2009). http://www.math.u-psud.fr/ illusie/refined_ uniformization3.pdf

11. Milne, J.S.: Lectures on étale cohomology (2013). http://www.jmilne.org/math/CourseNotes/lec.html

12. Pereira, J.V.: Fibrations, divisors and transcendental leaves. J. Algebraic Geom. 15(1), 87-110 (2006)

13. Silberstein, A.M.: Anabelian Intersection Theory. PhD thesis, Harvard University. ProQuest LLC, Ann Arbor (2012)

14. Seidenberg, A.: The hyperplane sections of normal varieties. Trans. Amer. Math. Soc. 69(2), 357-386 (1950)

15. Totaro, B.: The topology of smooth divisors and the arithmetic of abelian varieties. Michigan Math. J. 48, 611-624 (2000) 